\title{
Elevated Intraocular Pressure in Burn Patients Undergoing Fluid Resuscitation: An Opportunity to Avoid Orbital Compartment Syndrome
}

\author{
Christine Y. Shen, BS ${ }^{1}$ Spencer Woody, BS ${ }^{2}$ Spencer D. Fuller, MD, MPH ${ }^{1} \quad$ Landon K. Grange, MD ${ }^{1}$ \\ Kimberly D. Tran, MD ${ }^{1,4}$ Jeanne G. Lee, MD, FACS ${ }^{3}$ Don O. Kikkawa, MD, FACS ${ }^{1}$ Jeffrey E. Lee, MD ${ }^{1}$
}

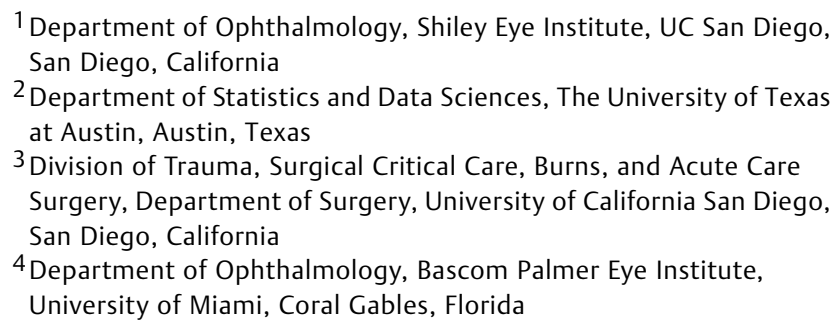

Address for correspondence Jeffrey E. Lee, MD, Department of Ophthalmology, Shiley Eye Institute, University of California San Diego, 200 West Arbor Drive no. 8655, San Diego, CA 92103-8655 (e-mail: j139lee@ucsd.edu).

J Acad Ophthalmol 2020;12:e87-e91.

\author{
Abstract \\ Keywords \\ - elevated intraocular \\ pressure \\ - orbital compartment \\ syndrome \\ - orbital \\ decompression \\ - fluid resuscitation \\ - parkland formula \\ - burn
}

Objective This study aims to discuss clinical characteristics of burn patients who developed elevated intraocular pressure (IOP). We propose management strategies to prevent orbital compartment syndrome before invasive treatment is required.

Methods A retrospective review of 47 burn patients at the University of California San Diego (UCSD), Burn Intensive Care Unit (ICU; major regional burn center for San Diego county), was analyzed for demographics, fluid resuscitation volumes, and physical exam findings. Patients requiring topical treatment for high-IOP, defined as $\geq 30 \mathrm{~mm} \mathrm{Hg}$ in either or both eyes, were compared with those who did not require treatment, using the $t$-test and Fisher's exact test. Linear regression tested for an association between peak IOP and fluid volume. Logistic regression evaluated the association between total fluid and treatment of high IOP, while adjusting for other characteristics.

Results Six of 47 patients required IOP-lowering treatment. Of the patients requiring treatment, one drop of dorzolamide/timolol in both eyes twice daily was the most common treatment recommendation. Presence of periocular burns was a significant risk factor in patients who required treatment for high IOP. No patients developed orbital compartment syndrome or required surgical intervention to lower IOP. The mean total fluid volume delivered in the first 24 hours was 0.8 times the level prescribed by fluid resuscitation guidelines.

Conclusion By treating early clinical signs and properly managing fluid resuscitation, development of orbital compartment syndrome in burn patients can be avoided.
Intraorbital pressures, reflected by intraocular pressure (IOP) measurements, rapidly rise regardless of etiology if swelling of the orbital soft tissues takes place. This can lead to complications including orbital compartment syndrome(OCS), which is similar to the condition described in abdominal compartment syndrome and compartment syndrome of the extremities in orthopaedic cases. ${ }^{1-5}$ Orbital compartment syndrome is a sight-threatening emergency and can rapidly lead to received

January 9, 2020

accepted

April 23, 2020
DOI https://doi.org/

10.1055/s-0040-1713146. ISSN 2475-4757.
Copyright $\odot 2020$ by Thieme Medical Publishers, Inc., 333 Seventh Avenue, New York, NY 10001, USA. Tel: +1(212) 760-0888.
License terms

(요 (1) $\Theta \circledast$ 
irreversible vision loss. Several mechanisms have been proposed, though no clear etiology has been proven. ${ }^{1}$ The orbit is a nonexpandable space, surrounded by four bony walls superiorly, inferiorly, medially, and laterally and a thick, fibrous orbital septum anteriorly. Vision loss due to OCS is thought to be caused by elevated orbital pressure leading to occlusion of posterior ciliary arteries, decreasing perfusion to the retina and optic nerve. ${ }^{6}$

Previous literature has suggested an association between orbital compartment syndrome and severe burn patients who require large volume fluid resuscitation, which we define as greater than two times the fluid volume recommended by the standard fluid resuscitation formula. ${ }^{7-10}$ In one study, nearly one-third of patients, with $\geq 25 \%$ total body surface area (TBSA) burnt, required emergent surgical orbital decompression. ${ }^{11}$ In several studies, justification of such treatment was supported by findings of significant vision loss following severe burn and trauma. ${ }^{1,2,12}$

Fluid resuscitation is a cornerstone of acute burn injury management. Since its conception in 1968, the Parkland formula, also known as the Baxter formula, has helped guide clinicians in delivering acute resuscitative fluids. This formula was based on the observation in both canine models and a pilot human population, that one could restore cardiac output, extracellular fluid, and plasma volumes to near normal by delivering intravenous (IV) fluids at a volume of $4.3 \mathrm{~mL} / \mathrm{kg} / \%$ TBSA over the first 24 hours after thermal injury. ${ }^{7}$ While many variations have been described, the Baxter formula remains the most commonly cited fluid resuscitation guideline and has markedly decreased rates of mortality previously attributed to burn shock, poor perfusion, and acute renal injury.

Recently, however, several studies have cited an increased incidence of resuscitation with fluids that exceed the recommended volume calculated from the Baxter formula, in a phenomenon termed "fluid creep." 13 There are several hypothesized reasons described by Saffle including overresuscitation by inexperienced health providers, overestimates of \% TBSA involved, and unrestricted administration of IV fluids in triage prior to admission to a burn center. ${ }^{13,14}$ Furthermore, modern iterations of the Baxter formula have omitted use of colloid, which was an original component of Baxter's formula in 1968 when he found that the use of a plasma bolus at 24 hours postinjury was helpful in restoring extracellular fluid balance. ${ }^{13}$ This is potentially problematic because increased absolute volumes of IV fluid resuscitation have been correlated with a rising incidence of edema-related complications including pericardial effusions, compartmental compression in unburned extremities, abdominal compartment syndrome, and elevated IOPs. ${ }^{4,11,15}$

In our experience at a major regional burn center, we have taken care of burn patients who developed elevated IOPs but found that surgical intervention is rarely indicated. The goal of this study is to better characterize the risk factors associated with elevated IOP, including fluid resuscitation, and to discuss possible reasons why none of our patients developed orbital compartment syndrome.

\section{Methods}

We retrospectively reviewed the medical records of burn patients from July 2008 to June 2017 after receiving approval from the University of California San Diego (UCSD) Human Research Protections Program Institutional Review Board. All patients were treated at the UCSD Burn Intensive Care Unit (ICU). Patients $\geq 18$ years of age with $\geq 25 \%$ TBSA burn were included. All patients included in the review underwent fluid resuscitation as calculated by the Baxter formula. Any patients with orbital trauma, hyphema, glaucoma, thirddegree periorbital burns, or chronic intraocular-lowering medications were excluded from the study. Thus, 47 patients met study criteria.

Evaluation of pertinent medical data included eye exam findings, past history, age, sex, race, past medications, concurrent medications and health problems, radiographic images, treatment, and subsequent clinical course as documented in the patient chart. We also recorded readmission, death, and posttreatment status of all patients at the 3-day end point.

Additionally, the UCSD Burn ICU meticulously kept track of fluid resuscitation for each burn patient. In every case, the stated goal was to follow the Baxter formula for fluid resuscitation. The recommended fluid volume from the Baxter formula was calculated for every patient based on their admission weights and TBSA percentages. The actual amount of fluids delivered to the patient were then compared with the amount prescribed by the Baxter formula.

Periocular burns were defined as burns involving the eyelids. Mortality was defined as failure to survive to hospital discharge. Peak IOP was defined as the single highest IOP measured with a commercially available Tonopen (Reichert XL, Depew, NY). All IOP measurements were recorded in $\mathrm{mm}$ $\mathrm{Hg}$ per eye per patient. The highest single IOP value was recorded for a patient's right and left eyes, respectively, during the course of hospital stay. These values were not necessarily obtained on the same day for a given patient, but rather represent the single highest IOP value obtained over the course of the patient's hospitalization.

Peak IOP and fluid levels were log transformed to reduce skewness. Linear regression was used to evaluate whether there was an association between log-peak IOP and fluid volume as measured by both total fluid $(\mathrm{mL})$ and $\mathrm{mL} / \mathrm{kg} / \%$ TBSA burned. Logistic regression was used to control for other characteristics when evaluating the association between total fluid and treatment of high IOP. Continuous variables were compared using a two-sided $t$-test and categorical variables were compared using a two-sided Fisher's exact test. Tests were conducted at the $\alpha=0.05$ significance level. Patient data were analyzed using the R programming language (v. 3.5.1).

\section{Results}

- Table 1 compares the demographics and clinical characteristics of the 47 patients included in this study. The \% TBSA ranged from 25 to $85 \%$ (mean, $45 \%$ ). In every case, the Baxter formula guided fluid resuscitation. No patient developed a 
Table 1 Comparison of demographics and clinical characteristics of patients who were treated for high-intraocular pressure versus those who were not

\begin{tabular}{|c|c|c|c|c|c|}
\hline \multirow[t]{2}{*}{ Characteristic } & \multicolumn{2}{|c|}{ No treatment $(n=41)$} & \multicolumn{2}{|c|}{$\begin{array}{l}\text { Treated for high intraocular pres- } \\
\text { sure }(n=6)\end{array}$} & \multirow[t]{2}{*}{$p^{a}$} \\
\hline & Range or \% & Mean \pm SE & Range or \% & Mean \pm SE & \\
\hline Age $(y)$ & $20-76$ & $46.0 \pm 2.46$ & $21-62$ & $46.7 \pm 6.63$ & 0.925 \\
\hline Weight $(\mathrm{kg})$ & $50-136$ & $86.1 \pm 3.35$ & $63.4-110$ & $78.1 \pm 6.89$ & 0.3282 \\
\hline Peak IOP $(\mathrm{mm} \mathrm{Hg})$ & $8-29$ & $18.6 \pm 0.709$ & $21.5-37$ & $30.2 \pm 2.30$ & 0.003 \\
\hline \% female & 34.1 & $\mathrm{~N} / \mathrm{A}$ & 33.3 & $\mathrm{~N} / \mathrm{A}$ & $1^{\mathrm{b}}$ \\
\hline \% periocular burn & 26.8 & $\mathrm{~N} / \mathrm{A}$ & 83.3 & $\mathrm{~N} / \mathrm{A}$ & $0.01336^{\mathrm{b}}$ \\
\hline$\%$ mortality & 4.9 & $\mathrm{~N} / \mathrm{A}$ & 16.7 & $\mathrm{~N} / \mathrm{A}$ & $0.3426^{\mathrm{b}}$ \\
\hline $\begin{array}{l}\text { Baxter formula, predicted } \\
\text { fluid }(\mathrm{mL}), 24 \mathrm{~h}\end{array}$ & $6,026-30,696$ & $15,785 \pm 1,011$ & $1,886-29,240$ & $15,442 \pm 3,766$ & 0.933 \\
\hline Total fluid, $24 \mathrm{~h}(\mathrm{~mL})$ & $1,738-37,254$ & $11,597 \pm 1,210$ & $4,964-35,883$ & $19,763 \pm 4,569$ & 0.1372 \\
\hline Total fluid, $48 \mathrm{~h}(\mathrm{~mL})$ & $3,000-31,706$ & $16,369 \pm 1,138$ & $7,206-57,196$ & $26,909 \pm 6,974$ & 0.1931 \\
\hline Total fluid, $72 \mathrm{~h}$ & $3,812-39,137$ & $13,912 \pm 1,469$ & $7,834-81,758$ & $32,928 \pm 11,314$ & 0.1545 \\
\hline Fluid, $24 \mathrm{~h}, \mathrm{~mL} / \mathrm{kg} / \%$ TBSA burned & $54.6-1,067$ & $339 \pm 35.9$ & $196-1,071$ & $489 \pm 132$ & 0.3155 \\
\hline Fluid, $48 \mathrm{~h}, \mathrm{~mL} / \mathrm{kg} / \%$ TBSA burned & $127-1,643$ & $520 \pm 52.8$ & $106-1,177$ & $711 \pm 168$ & 0.3202 \\
\hline Fluid, $72 \mathrm{~h}, \mathrm{~mL} / \mathrm{kg} / \%$ TBSA burned & $62.9-1,276$ & $427 \pm 52.1$ & $124-1,775$ & $889 \pm 291$ & 0.1745 \\
\hline
\end{tabular}

Abbreviations: IOP, intraocular pressure; N/A, not available; TBSA, total body surface area; SE, standard error.

${ }^{a} p$ from $t$-test.

b $p$ from Fisher's exact test.

relative afferent pupillary defect, and none had additional evidence of optic nerve compromise such as impaired color vision.

No patients developed orbital compartment syndrome or required surgical intervention during or after their course of hospitalization. Therefore, we assessed clinical differences among burn patients who required treatment for elevated IOP, defined as $\geq 30 \mathrm{~mm} \mathrm{Hg}$, and those who did not. The peak IOP ranged from 8 to $37 \mathrm{~mm} \mathrm{Hg}$ (mean, $20 \mathrm{~mm} \mathrm{Hg}$ ).

Of the 47 total patients, only six patients had IOPs high enough to warrant treatment with IOP-lowering medications. The peak IOP of the treatment group was 21.5 to $37 \mathrm{~mm} \mathrm{Hg}$ (mean, $30.2 \mathrm{~mm} \mathrm{Hg}$ ) versus 8 to $29 \mathrm{~mm} \mathrm{Hg}$ (mean, $18.6 \mathrm{~mm} \mathrm{Hg}$ ). The difference in peak IOP between the two groups was statistically significant $(p=0.003)$.

In addition, presence of periocular burns was a significant risk factor in patients who required treatment for high IOP $(p=0.001)$. Periocular burns were categorized into presence or absence of periocular burns for analysis. Concerning the extent of injury, 16 patients (34\%) had second-degree eyelid burns, with the vast majority being bilateral; 15 (32\%) had singed lashes, but no second-degree burns. The remaining patients who sustained periocular burns had varying degrees of facial involvement and/or eyelid edema from third spacing. No patients sustained third-degree burns of the periorbita.

The patients who required treatment, five out of six were treated with one drop of combination dorzolamide/timolol (Cosopt) in both eyes twice a day. One of these five patients had IOPs on admission of 36 and $38 \mathrm{~mm} \mathrm{Hg}$ and was started on brimonidine tartrate three times a day in both eyes in addition to combination dorzolamide/timolol. Within 12 hours, the IOP had decreased to $<30 \mathrm{~mm} \mathrm{Hg}$ in both eyes, at which point brimonidine tartrate was stopped. The patient was continued on combination dorzolamide/timolol twice a day for a total of 48 hours until the IOPs came down to 7 and $9 \mathrm{~mm} \mathrm{Hg}$. The sixth patient was prescribed one drop of latanoprost in both eyes at bedtime for one day.

The relative fluid resuscitation volumes were assessed for differences between the patient group treated for high IOP and the group that was not treated and are included in - Table $\mathbf{1}$. The amount of fluid recommended by the Baxter formula at 24 hours was not statistically different between the two groups $(p=0.933)$. Total volume of fluid administered at 24,48 , and 72 hours were also not statistically significant between the two groups. The total amount of fluid delivered at 24 hours was higher in the group that required treatment, $19,763 \pm 4,569$ versus $11,597 \pm 1,210 \mathrm{~mL}$. This was not statistically significant $(p=0.1372)$, even after adjusting for other key covariates $(p=0.07 ;-$ Table 2). However, logistic regression analysis found a statistically significant association between risk of treatment for high IOP and periocular burns $(p=0.04)$.

Linear regression analysis found no significant correlation between peak IOP in the first 48 hours and the fluid volume administered at 24 hours, both in total $\mathrm{ml}\left(r^{2}=0.026\right.$, $p=0.282)$ and $\mathrm{mL} / \mathrm{kg} / \% \mathrm{TBSA}\left(r^{2}=0.041, p=0.177\right)$. There were no statistically significant differences between the groups in terms of age, sex, weight, or mortality.

The mean predicted volume of fluid resuscitation delivered, derived from the Baxter equation ( $4.3 \mathrm{~mL} / \mathrm{kg} / \%$ TBSA burned), was $15,741 \pm 986 \mathrm{~mL}$. The mean total volume delivered in the first 24 hours was $12,662 \pm 1,254$ or $356 \pm 35.8 \mathrm{~mL} / \mathrm{kg} / \%$ TBSA burned ( 0.8 times the level prescribed by the Baxter formula). 
Table 2 Association between total fluid at 24 hours $(\mathrm{mL}$, natural $\mathrm{log}$ ) and treatment of high-intraocular pressure, before and after adjusting for key covariates

\begin{tabular}{|l|l|}
\hline Covariate & $p^{\mathrm{a}}$ \\
\hline Total fluid, 24 $\mathrm{h}(\mathrm{mL})$, unadjusted & $0.1372^{\mathrm{b}}$ \\
\hline Total fluid, 24 h $(\mathrm{mL})$, adjusted & 0.0731 \\
\hline Age & 0.8459 \\
\hline Sex & 0.4145 \\
\hline Weight $(\mathrm{kg})$ & 0.1679 \\
\hline Sex and weight $(\mathrm{kg})$ & 0.3285 \\
\hline Periocular burn & 0.0356 \\
\hline
\end{tabular}

${ }^{a} p$ from logistic model for $\ln$ (fluid) and covariates.

${ }^{\mathrm{b}} p$ from $t$ test.

Roughly two-thirds of both groups received fluid resuscitation in accordance with the Baxter formula. Only one patient in the study exceeded double the amount prescribed by the Baxter formula at 24 hours but did not require treatment. Two others exceeded double the amount within the first 48 hours. One underwent hemodialysis to correct for overresuscitation, and the other died during hospitalization.

\section{Discussion}

Our results may offer insights on how to prevent the development of orbital compartment syndrome in severely burn patients with proper management of fluid resuscitation volumes. As a priority in our burn unit, the stated goal is to minimize deviations from the Baxter formula for fluid resuscitation. The mean total volume delivered in the first 24 hours was 0.8 times the level predicted by the Baxter formula. No patients developed orbital compartment syndrome during or after the course of hospitalization in our study. Few even required topical pressure lowering agents, and none required surgical intervention. Stricter adherence to the Baxter formula could potentially explain the absence of orbital compartment syndrome in our burn patients, though this hypothesis is suggestive and not conclusive. A previous study found that patients receiving more than twice the fluids recommended by the Baxter formula were 4.4 times as likely to develop severe orbital compartment syndrome. ${ }^{11}$ However, our results detected that fluid volume was not a significant risk factor for patients requiring topical IOP-lowering intervention. We believe that this could be due to the small sample size of our cohort and that few patients required such intervention which could have led to lower statistical power to replicate this link.

Only one patient received more than double the amount prescribed by the Baxter formula in the first 24 hours and did not require IOP-lowering treatment. In another case where the fluid resuscitation exceeded twice the Baxter formula within the first 48 hours, hemodialysis was initiated to correct for overresuscitation. The last patient who exceeded twice the Baxter formula in 48 hours expired during hospitalization. One possible limitation of this study was that data collected reflected total fluid volume replenishment and not the rate of fluid bolus in these patients. The rate of fluid administration could be a factor in the development of orbital compartment syndrome, but our study focused on total volume replacement.

The management of OCS has been well described. ${ }^{1,16-19}$ IOP and vision may be fully restored to baselines following prompt diagnosis and treatment. Medical management includes use of steroids, carbonic anhydrase inhibitors, and mannitol. In emergent cases, lateral canthotomy and cantholysis and/or orbital decompression can surgically reduce intraorbital pressure. Return of vision has been reported after decompression suggesting return of perfusion. 1,16,19,20 Studies have shown immediate decrease in intraorbital pressure after canthotomy and cantholysis, and case reports have demonstrated the efficacy of this procedure to restore vision. ${ }^{21-24}$ If the patient fails to show improvement, the orbit may be further decompressed by opening of the orbital septum and/or bony expansion of the orbit by removing one of the orbital walls.

In this study, we did not find the need for surgical options such as canthotomy and cantholysis and orbital decompression. The presence of periocular burns, however, was a significant risk factor associated with elevated IOP requiring pressure lowering medication $(p=0.003)$. A total of $83.3 \%$ of patients who required treatment to lower IOP had periocular burns compared with $26.8 \%$ of those who did not ( - Table 1 ). Logistic regression analyses also found a statistically significant association between treatment of high IOP and presence of periocular burns ( $p=0.04$; - Table 2 ). This suggests that periocular burns could play a significant role in increasing IOP, which may be a reflection of underlying high intraorbital pressure or a primary globe side effect of the thermal injury. Five out of six patients who required treatment received one drop of combination dorzolamide/timolol (Cosopt) in both eyes twice a day. Within their hospital stay, all patients had IOPs less than $20 \mathrm{~mm} \mathrm{Hg}$ with topical treatment. In our opinion, topical treatment of high IOPs could be considered part of standard ophthalmic management in these patients and may be related to globe trauma and anterior lid involvement, rather than reflecting deeper impending orbital compartment syndrome.

\section{Conclusion}

It is important to note the relative geographic and population size differences between regional burn centers. The UCSD Regional Burn Center may have a different population than centers with higher percentages of orbital compartment syndrome. These burn centers may have larger geographic catchment areas and rural locales from which they accept patients. Since the first 24 hours of fluid resuscitation is of paramount importance, it is possible that a higher relative reliance on triage providers and emergency medical personnel unfamiliar with the Baxter protocol may account for some of the observed differences. Future studies may explore whether this association may hold true. The results of this study show promise that orbital compartment syndrome can be prevented with strict fluid replenishment, close surveillance, and treatment of ocular issues early in the treatment course. 


\section{Conflict of Interest \\ None declared.}

\section{References}

1 Lima V, Burt B, Leibovitch I, Prabhakaran V, Goldberg RA, Selva D. Orbital compartment syndrome: the ophthalmic surgical emergency. Surv Ophthalmol 2009;54(04):441-449

2 Klein MB, Hayden D, Elson C, et al. The association between fluid administration and outcome following major burn: a multicenter study. Ann Surg 2007;245(04):622-628

3 Oda J, Yamashita K, Inoue T, et al. Resuscitation fluid volume and abdominal compartment syndrome in patients with major burns. Burns 2006;32(02):151-154

4 Ivy ME, Atweh NA, Palmer J, Possenti PP, Pineau M, D’Aiuto M. Intra-abdominal hypertension and abdominal compartment syndrome in burn patients. J Trauma 2000;49(03):387-391

5 Perron AD, Brady WJ, Keats TE. Orthopedic pitfalls in the ED: acute compartment syndrome. Am J Emerg Med 2001;19(05):413-416

6 Goodall KL, Brahma A, Bates A, Leatherbarrow B. Lateral canthotomy and inferior cantholysis: an effective method of urgent orbital decompression for sight threatening acute retrobulbar haemorrhage. Injury 1999;30(07):485-490

7 Baxter CR, Shires T. Physiological response to crystalloid resuscitation of severe burns. Ann N Y Acad Sci 1968;150(03):874-894

8 Engrav LH, Colescott PL, Kemalyan N, et al. A biopsy of the use of the Baxter formula to resuscitate burns or do we do it like Charlie did it? J Burn Care Rehabil 2000;21(02):91-95

9 Friedrich JB, Sullivan SR, Engrav LH, et al. Is supra-Baxter resuscitation in burn patients a new phenomenon? Burns 2004;30(05):464-466

10 Cancio LC, Chávez S, Alvarado-Ortega M, et al. Predicting increased fluid requirements during the resuscitation of thermally injured patients. J Trauma 2004;56(02):404-413, discussion 413-414

11 Singh CN, Klein MB, Sullivan SR, et al. Orbital compartment syndrome in burn patients. Ophthal Plast Reconstr Surg 2008;24(02):102-106

12 Sullivan SR, Ahmadi AJ, Singh CN, et al. Elevated orbital pressure: another untoward effect of massive resuscitation after burn injury. J Trauma 2006;60(01):72-76
13 Saffle JI. The phenomenon of "fluid creep" in acute burn resuscitation. J Burn Care Res 2007;28(03):382-395

14 Saffle JR. Fluid Creep and Over-resuscitation. Crit Care Clin 2016; 32(04):587-598

15 Tekin A, Namias N, O'Keeffe T, et al. A burn mass casualty event due to boiler room explosion on a cruise ship: preparedness and outcomes. Am Surg 2005;71(03):210-215

16 Winterton JV, Patel K, Mizen KD. Review of management options for a retrobulbar hemorrhage. J Oral Maxillofac Surg 2007;65(02): 296-299

17 Vassallo S, Hartstein M, Howard D, Stetz J. Traumatic retrobulbar hemorrhage: emergent decompression by lateral canthotomy and cantholysis. J Emerg Med 2002;22(03):251-256

18 Ballard SR, Enzenauer RW, O'Donnell T, Fleming JC, Risk G, Waite AN. Emergency lateral canthotomy and cantholysis: a simple procedure to preserve vision from sight threatening orbital hemorrhage. J Spec Oper Med 2009;9(03):26-32

19 Rowh AD, Ufberg JW, Chan TC, Vilke GM, Harrigan RA. Lateral canthotomy and cantholysis: emergency management of orbital compartment syndrome. J Emerg Med 2015;48(03): 325-330

20 Kitaguchi Y, Takahashi Y, Sabundayo MS, Kakizaki H. Bony orbital decompression following lateral canthotomy and cantholysis for traumatic orbital compartment syndrome. J Craniofac Surg 2019; 30(01):231-234

21 Rosdeutscher JD, Stadelmann WK. Diagnosis and treatment of retrobulbar hematoma resulting from blunt periorbital trauma. Ann Plast Surg 1998;41(06):618-622

22 Yung C-W, Moorthy RS, Lindley D, Ringle M, Nunery WR. Efficacy of lateral canthotomy and cantholysis in orbital hemorrhage. Ophthal Plast Reconstr Surg 1994;10(02):137-141

23 Krausen AS, Ogura JH, Burde RM, Ostrow DE. Emergency orbital decompression: a reprieve from blindness. Otolaryngol Head Neck Surg 1981;89(02):252-256

24 Prodhan P, Noviski NN, Butler WE, Eskandar E, Ellen Grant P, Whalen MJ. Orbital compartment syndrome mimicking cerebral herniation in a 12-yr-old boy with severe traumatic asphyxia. Pediatr Crit Care Med 2003;4(03):367-369 